\title{
QUENCHING FOR A DEGENERATE PARABOLIC PROBLEM DUE TO A CONCENTRATED NONLINEAR SOURCE
}

BY

\author{
C. Y. CHAN AND X. O. JIANG
}

Department of Mathematics, University of Louisiana at Lafayette, Lafayette, Louisiana 70504-1010

Abstract. Let $q, a, T$, and $b$ be any real numbers such that $q \geq 0, a>0, T>0$, and $0<b<1$. This article studies the following degenerate semilinear parabolic first initial-boundary value problem with a concentrated nonlinear source situated at $b$ :

$$
\begin{gathered}
x^{q} u_{t}-u_{x x}=a^{2} \delta(x-b) f(u(x, t)) \text { in }(0,1) \times(0, T], \\
u(x, 0)=0 \text { on }[0,1], u(0, t)=u(1, t)=0 \text { for } 0<t \leq T,
\end{gathered}
$$

where $\delta(x)$ is the Dirac delta function, $f$ is a given function such that $\lim _{u \rightarrow c^{-}} f(u)=\infty$ for some positive constant $c$, and $f(u)$ and $f^{\prime}(u)$ are positive for $0 \leq u<c$. It is shown that the problem has a unique continuous solution $u$ before $\max \{u(x, t): 0 \leq x \leq 1\}$ reaches $c^{-}, u$ is a strictly increasing function of $t$ for $0<x<1$, and if $\max \{u(x, t)$ : $0 \leq x \leq 1\}$ reaches $c^{-}$, then $u$ attains the value $c$ only at the point $b$. The problem is shown to have a unique $a^{*}$ such that a unique global solution $u$ exists for $a \leq a^{*}$, and $\max \{u(x, t): 0 \leq x \leq 1\}$ reaches $c^{-}$in a finite time for $a>a^{*}$; this $a^{*}$ is the same as that for $q=0$. A formula for computing $a^{*}$ is given, and no quenching in infinite time is deduced.

1. Introduction. Let $q, \beta, a$, and $\rho$ be any real numbers with $q \geq 0,0<\beta<a$, and $\rho>0$. Let us consider the following degenerate semilinear parabolic first initial-boundary value problem,

$$
\left.\begin{array}{c}
\varsigma^{q} u_{\gamma}-u_{\varsigma \varsigma}=\delta(\varsigma-\beta) F(u(\varsigma, \gamma)) \text { in }(0, a) \times(0, \rho], \\
u(\varsigma, 0)=0 \text { on }[0, a], u(0, \gamma)=u(a, \gamma)=0 \text { for } 0<\gamma \leq \rho,
\end{array}\right\}
$$

where $\delta(x)$ is the Dirac delta function and $F$ is a given function. These types of problems are motivated by applications in which the ignition of a combustible medium is accomplished through the use of either a heated wire or a pair of small electrodes to supply a large amount of energy to a very confined area. When $q=0$, the problem

Received July 7, 2003.

2000 Mathematics Subject Classification. Primary 35K60, 35K65, 35K57.

Key words and phrases. Degenerate parabolic problem, concentrated nonlinear source, unique continuous solution, single-point quenching, critical length, no quenching in infinite time.

E-mail address: chan@louisiana.edu

E-mail address: xoj8985@louisiana.edu 
(1) can be used to describe the temperature of a one-dimensional rod having a length $a$ and a concentrated nonlinear source at $\beta$. When $q=1$, it may also be used to describe the temperature $u$ of the channel flow of a fluid with temperature-dependent viscosity in the boundary layer (cf. Chan and Kong [4]) with a concentrated nonlinear source at $\beta$; here, $\varsigma$ and $\gamma$ denote the coordinates perpendicular and parallel to the channel wall, respectively.

Let $\varsigma=a x, \gamma=a^{q+2} t, \beta=a b, L u=x^{q} u_{t}-u_{x x}, F(u(\varsigma, \gamma))=f(u(x, t)), D=(0,1)$, $\bar{D}=[0,1]$, and $\Omega=D \times(0, T]$. Then (1) is transformed into the following problem:

$$
\left.\begin{array}{c}
L u=a^{2} \delta(x-b) f(u(x, t)) \text { in } \Omega, \\
u(x, 0)=0 \text { on } \bar{D}, u(0, t)=u(1, t)=0 \text { for } 0<t \leq T,
\end{array}\right\}
$$

with $0<b<1$ and $T=\rho / a^{q+2}$. We assume that $\lim _{u \rightarrow c^{-}} f(u)=\infty$ for some positive constant $c$, and $f(u)$ and $f^{\prime}(u)$ are positive for $0 \leq u<c$.

The case $q=0$ was studied by Deng and Roberts [7] by analyzing its corresponding nonlinear Volterra equation at the site $b$ of the concentrated source:

$$
u(b, t)=a^{2} \int_{0}^{t} g(b, t ; b, \tau) f(u(b, \tau)) d \tau,
$$

where $g(x, t ; \xi, \tau)$ denotes Green's function corresponding to (2) with $q=0$. By also assuming that $f^{\prime \prime}(u)>0$ for $u \geq 0$, they showed that there exists a length $a^{*}$ such that for $a \leq a^{*}$, the solution $u(b, t)$ of the integral equation exists for all time and is uniformly bounded away from $c$ while for $a>a^{*}$, there exists some finite $t_{q}$ such that $\lim _{t \rightarrow t_{q}} u(b, t)=c$ and $\lim _{t \rightarrow t_{q}} u_{t}(b, t)=\infty$.

Instead of studying a solution $u(b, t)$ of the nonlinear Volterra equation, we would like to investigate a solution $u(x, t)$ of the degenerate problem (2). Since $u(x, t)$ need not be differentiable at $b$, we say that a solution of the problem (2) is a continuous function satisfying (2). In Sec. 2, we show that the problem (2) has a unique solution $u$, and $u_{x x} \geq$ 0 for $x \in(0, b)$ and $x \in(b, 1)$. It follows from $x^{q} u_{t}(x, t)=u_{x x}(x, t)+a^{2} \delta(x-b) f(u(x, t))$ that $u_{t}(b, t)=\infty$ for each $t>0$. Hence, we say that a solution $u$ of the problem (2) is said to quench if there exists some $t_{q}$ such that $\max \{u(x, t): x \in \bar{D}\} \rightarrow c^{-}$as $t \rightarrow t_{q}$ (cf. Chan and Liu [5]). If $t_{q}$ is finite, then $u$ is said to quench in a finite time. On the other hand, if $t_{q}=\infty$, then $u$ is said to quench in infinite time. We also show that $u$ is a strictly increasing function of $t$ in $D$, and if $u$ quenches, then $b$ is the single quenching point.

The length $a^{*}$ is called the critical length (cf. Chan and Kong [3]) if a unique global solution $u$ exists for $a<a^{*}$, and if the solution $u$ quenches in a finite time for $a>a^{*}$. In Sec. 3, we show existence of a unique critical length, and that it is the same as that for $q=0$. By making use of $\lim _{u \rightarrow c^{-}} f(u)=\infty$, we show that for $a=a^{*}, u$ exists for $0<t<\infty$, and is uniformly bounded away from $c$. This shows that quenching does not occur in infinite time. We also derive a formula for calculating $a^{*}$.

2. Existence, uniqueness, and single-point quenching. Green's function $G(x, t ; \xi, \tau)$ corresponding to the problem (2) is determined by the following system: 
for $x$ and $\xi$ in $D$, and $t$ and $\tau$ in $(-\infty, \infty)$,

$$
\begin{gathered}
L G=\delta(x-\xi) \delta(t-\tau), \\
G(x, t ; \xi, \tau)=0 \text { for } t<\tau, G(0, t ; \xi, \tau)=G(1, t ; \xi, \tau)=0 .
\end{gathered}
$$

By Chan and Chan [1], we have

$$
G(x, t ; \xi, \tau)=\sum_{i=1}^{\infty} \phi_{i}(x) \phi_{i}(\xi) e^{-\lambda_{i}(t-\tau)},
$$

where $\lambda_{i}(i=1,2,3, \ldots)$ are the eigenvalues of the problem

$$
\phi^{\prime \prime}+\lambda x^{q} \phi=0, \phi(0)=\phi(1)=0,
$$

and their corresponding eigenfunctions are given by

$$
\phi_{i}(x)=(q+2)^{1 / 2} x^{1 / 2} \frac{J_{\frac{1}{q+2}}\left(\frac{2 \lambda_{i}^{1 / 2}}{q+2} x^{(q+2) / 2}\right)}{\left|J_{1+\frac{1}{q+2}}\left(\frac{2 \lambda_{i}^{1 / 2}}{q+2}\right)\right|}
$$

with $J_{1 /(q+2)}$ denoting the Bessel function of the first kind of order $1 /(q+2)$. From Chan and Chan [1], $0<\lambda_{1}<\lambda_{2}<\lambda_{3}<\cdots<\lambda_{i}<\lambda_{i+1}<\cdots$. The set $\left\{\phi_{i}(x)\right\}$ is a maximal (that is, complete) orthonormal set with the weight function $x^{q}$ (cf. Gustafson $[9$, p. 176]).

To derive the integral equation from the problem (2), let us consider the adjoint operator $L^{*}$, which is given by $L^{*} u=-x^{q} u_{t}-u_{x x}$. Using Green's second identity, we obtain

$$
u(x, t)=a^{2} \int_{0}^{t} G(x, t ; b, \tau) f(u(b, \tau)) d \tau .
$$

For ease of reference, let us state below Lemmas 1(a), 1(b), 1(d), and 4 of Chan and Chan [1] as Lemma 1(a), 1(b), 1(c), and 1(d), respectively; we also state below Lemma 2.2(a), 2.2(b), 2.2(c), and 2.2(d) of Chan and Tian [6] as Lemma 1(e), 1(f), 1(g), and $1(\mathrm{~h})$, respectively.

Lemma 1. (a). For some positive constant $k_{1},\left|\phi_{i}(x)\right| \leq k_{1} x^{-q / 4}$ for $x \in(0,1]$.

(b). For some positive constant $k_{2},\left|\phi_{i}(x)\right| \leq k_{2} x^{1 / 2} \lambda_{i}^{1 / 4}$ for $x \in \bar{D}$.

(c). For any $x_{0}>0$, and $x \in\left[x_{0}, 1\right]$, there exists some positive constant $k_{3}$ depending on $x_{0}$ such that $\left|\phi_{i}^{\prime}(x)\right| \leq k_{3} \lambda_{i}^{1 / 2}$.

(d). In $\{(x, t ; \xi, \tau): x$ and $\xi$ are in $D, T \geq t>\tau \geq 0\}, G(x, t ; \xi, \tau)$ is positive.

(e). For $(x, t ; \xi, \tau) \in(\bar{D} \times(\tau, T]) \times(\bar{D} \times[0, T)), G(x, t ; \xi, \tau)$ is continuous.

(f). For each fixed $(\xi, \tau) \in \bar{D} \times[0, T), G_{t}(x, t ; \xi, \tau) \in C(\bar{D} \times(\tau, T])$.

(g). For each fixed $(\xi, \tau) \in \bar{D} \times[0, T), G_{x}(x, t ; \xi, \tau)$ and $G_{x x}(x, t ; \xi, \tau)$ are in $C((0,1]$ $\times(\tau, T])$.

(h). If $r \in C([0, T])$, then $\int_{0}^{t} G(x, t ; b, \tau) r(\tau) d \tau$ is continuous for $x \in \bar{D}$ and $t \in[0, T]$.

We modify the techniques in proving Lemma 2.3 and Theorems 2.4 and 2.6 of Chan and Tian [6] to show that the integral equation (3) has a unique nonnegative continuous solution. Unlike theirs, we achieve this without using the contraction mapping and 
without considering the integral equation (3) at $x=b$; also by making use of (3), we prove further that $u$ is a strictly increasing function of $t$ in $D$.

THEOREM 1. There exists some $t_{q}(\leq \infty)$ such that for $0 \leq t<t_{q}$, the integral equation (3) has a unique nonnegative continuous solution $u$, and $u$ is a strictly increasing function of $t$ in $D$. If $t_{q}$ is finite, then $u$ reaches $c^{-}$at $t_{q}$.

Proof. Let us construct a sequence $\left\{u_{i}\right\}$ in $\Omega$ by $u_{0} \equiv 0$, and for $i=0,1,2, \ldots$,

$$
\begin{aligned}
L u_{i+1} & =a^{2} \delta(x-b) f\left(u_{i}(x, t)\right) \text { in } \Omega, \\
u_{i+1}(x, 0) & =0 \text { on } \bar{D}, u_{i+1}(0, t)=u_{i+1}(1, t)=0 \text { for } 0<t \leq T .
\end{aligned}
$$

Let $\partial \Omega$ denote the parabolic boundary $(\bar{D} \times\{0\}) \cup(\{0,1\} \times(0, T])$ of $\Omega$. We have

$$
L\left(u_{1}-u_{0}\right)=a^{2} \delta(x-b) f(0) \text { in } \Omega, u_{1}-u_{0}=0 \text { on } \partial \Omega .
$$

By Lemma $1(\mathrm{~d})$ and $1(\mathrm{e}), G(x, t ; \xi, \tau)$ is positive and continuous. From $(3), u_{1}>u_{0}$ in $\Omega$. Let us assume that for some positive integer $j$,

$$
0<u_{1}<u_{2}<\cdots<u_{j-1}<u_{j} \text { in } \Omega \text {. }
$$

We have

$$
L\left(u_{j+1}-u_{j}\right)=a^{2} \delta(x-b)\left(f\left(u_{j}\right)-f\left(u_{j-1}\right)\right) \text { in } \Omega, u_{j+1}-u_{j}=0 \text { on } \partial \Omega .
$$

Since $f$ is a strictly increasing function and $u_{j}>u_{j-1}$, it follows from (3) that $u_{j+1}>u_{j}$. By the principle of mathematical induction,

$$
0<u_{1}<u_{2}<\cdots<u_{n-1}<u_{n} \text { in } \Omega
$$

for any positive integer $n$.

To show that each $u_{n}$ is an increasing function of $t$, let us construct a sequence $\left\{w_{n}\right\}$ such that for $n=0,1,2, \ldots, w_{n}(x, t)=u_{n}(x, t+h)-u_{n}(x, t)$, where $h$ is any positive number less than $T$. Then, $w_{0}(x, t)=0$. By $(3)$, we have

$$
\begin{aligned}
w_{1}(x, t) & =u_{1}(x, t+h)-u_{1}(x, t) \\
& =a^{2} f(0)\left(\int_{0}^{t+h} G(x, t+h ; b, \tau) d \tau-\int_{0}^{t} G(x, t ; b, \tau) d \tau\right) .
\end{aligned}
$$

We note that $G(x, t+h ; b, \tau)=G(x, t+h-\tau ; b, 0)$. Let $\sigma=\tau-h$. Then,

$$
\int_{0}^{t+h} G(x, t+h ; b, \tau) d \tau=\int_{0}^{h} G(x, t+h ; b, \tau) d \tau+\int_{0}^{t} G(x, t ; b, \sigma) d \sigma .
$$

We have

$$
w_{1}(x, t)=a^{2} f(0) \int_{0}^{h} G(x, t+h ; b, \tau) d \tau
$$


which is positive for $0<t \leq T-h$. Let us assume that for some positive integer $j$, $w_{j}>0$ for $0<t \leq T-h$. Let $\sigma=\tau-h$. We have

$$
\begin{aligned}
& a^{2} \int_{0}^{t+h} G(x, t+h ; b, \tau) f\left(u_{j}(b, \tau)\right) d \tau \\
& =a^{2}\left(\int_{0}^{h} G(x, t+h ; b, \tau) f\left(u_{j}(b, \tau)\right) d \tau+\int_{0}^{t} G(x, t ; b, \sigma) f\left(u_{j}(b, \sigma+h)\right) d \sigma\right) \\
& >a^{2}\left(\int_{0}^{h} G(x, t+h ; b, \tau) f\left(u_{j}(b, \tau)\right) d \tau+\int_{0}^{t} G(x, t ; b, \sigma) f\left(u_{j}(b, \sigma)\right) d \sigma\right) .
\end{aligned}
$$

In $D \times(0, T-h]$,

$$
\begin{aligned}
w_{j+1}(x, t) & =u_{j+1}(x, t+h)-u_{j+1}(x, t) \\
& >a^{2} \int_{0}^{h} G(x, t+h ; b, \tau) f\left(u_{j}(b, \tau)\right) d \tau>0
\end{aligned}
$$

By the principle of mathematical induction, $w_{n}>0$ for $0<t \leq T-h$ and all positive integers $n$. Thus, each $u_{n}$ is an increasing function of $t$.

By Lemma $1(\mathrm{~h}), G(x, t ; b, \tau)$ is integrable. Thus for any given positive constant $M$ $(<c)$, it follows from

$$
u_{n}(x, t)=a^{2} \int_{0}^{t} G(x, t ; b, \tau) f\left(u_{n-1}(b, \tau)\right) d \tau
$$

and $u_{n}$ being an increasing function of $t$ that there exists some $t_{1}$ such that $u_{n} \leq M$ for $0 \leq t \leq t_{1}$ and $n=0,1,2, \ldots$. In fact, $t_{1}$ satisfies

$$
a^{2} f(M) \int_{0}^{t_{1}} G\left(x, t_{1} ; b, \tau\right) d \tau \leq M
$$

Let $u$ denote $\lim _{n \rightarrow \infty} u_{n}$. From (4) and the Monotone Convergence Theorem (cf. Royden $\left[10\right.$, p. 87]), we have (3) for $0 \leq t \leq t_{1}$. Thus, $u$ is a nonnegative solution of the integral equation (3) for $0 \leq t \leq t_{1}$.

To prove that $u$ is unique, let us assume that the integral equation (3) has two distinct solutions $u$ and $\tilde{u}$ on the interval $\left[0, t_{1}\right]$. Let $\Theta=\max _{\bar{D} \times\left[0, t_{1}\right]}|u-\tilde{u}|$. From (3),

$$
u(x, t)-\tilde{u}(x, t)=a^{2} \int_{0}^{t} G(x, t ; b, \tau)(f(u(b, \tau))-f(\tilde{u}(b, \tau))) d \tau
$$

Using the Mean Value Theorem, we have

$$
|f(u(b, \tau))-f(\tilde{u}(b, \tau))| \leq f^{\prime}(M) \Theta
$$


Let $\epsilon$ be some positive number. It follows from Lemma 1(a), 1(b), and (5) that

$$
\begin{aligned}
\Theta & \leq a^{2} f^{\prime}(M) \Theta \int_{0}^{t} G(x, t ; b, \tau) d \tau \\
& \leq a^{2} f^{\prime}(M) \Theta k_{1} k_{2} b^{-q / 4} \lim _{\epsilon \rightarrow 0} \int_{0}^{t-\epsilon} \sum_{i=1}^{\infty} \lambda_{i}^{1 / 4} e^{-\lambda_{i}(t-\tau)} d \tau \\
& \leq a^{2} f^{\prime}(M) \Theta k_{1} k_{2} b^{-q / 4} \lim _{\epsilon \rightarrow 0} \int_{0}^{t-\epsilon} \sum_{i=1}^{\infty} \lambda_{i}^{1 / 4} e^{-\lambda_{i} \epsilon} d \tau
\end{aligned}
$$

Since $\sum_{i=1}^{\infty} \lambda_{i}^{1 / 4} e^{-\lambda_{i} \epsilon}$ converges uniformly with respect to $\tau \in[0, t-\epsilon]$, it follows from the Weierstrass M-Test that

$$
\begin{aligned}
\Theta & \leq a^{2} f^{\prime}(M) \Theta k_{1} k_{2} b^{-q / 4} \lim _{\epsilon \rightarrow 0} \sum_{i=1}^{\infty} \int_{0}^{t-\epsilon} \lambda_{i}^{1 / 4} e^{-\lambda_{i}(t-\tau)} d \tau \\
& \leq a^{2} f^{\prime}(M) \Theta k_{1} k_{2} b^{-q / 4} \lim _{\epsilon \rightarrow 0} \sum_{i=1}^{\infty} \lambda_{i}^{-3 / 4}\left(e^{-\lambda_{i} \epsilon}-e^{-\lambda_{i} t}\right) \\
& \leq a^{2} f^{\prime}(M) \Theta k_{1} k_{2} b^{-q / 4} \lim _{\epsilon \rightarrow 0} \sum_{i=1}^{\infty} \lambda_{i}^{-3 / 4}\left(1-e^{-\lambda_{i} t}\right) \\
& =a^{2} f^{\prime}(M) k_{1} k_{2} b^{-q / 4}\left[\sum_{i=1}^{\infty} \lambda_{i}^{-3 / 4}\left(1-e^{-\lambda_{i} t}\right)\right] \Theta
\end{aligned}
$$

which converges since $O\left(\lambda_{i}\right)=O\left(i^{2}\right)$ (cf. Watson [12, p. 506]) for large $i$. Let us choose some positive number $\sigma_{1}\left(\leq t_{1}\right)$ such that for $t \in\left[0, \sigma_{1}\right]$,

$$
a^{2} f^{\prime}(M) k_{1} k_{2} b^{-q / 4}\left[\sum_{i=1}^{\infty} \lambda_{i}^{-3 / 4}\left(1-e^{-\lambda_{i} t}\right)\right]<1 .
$$

This gives a contradiction. Thus, $u$ is unique for $0 \leq t \leq \sigma_{1}$. If $\sigma_{1}<t_{1}$, then it follows from (3) that for $\sigma_{1} \leq t \leq t_{1}$,

$$
u(x, t)=a^{2} \int_{0}^{\sigma_{1}} G(x, t ; b, \tau) f(u(b, \tau)) d \tau+a^{2} \int_{\sigma_{1}}^{t} G(x, t ; b, \tau) f(u(b, \tau)) d \tau .
$$

Since $u=\tilde{u}$ for $0 \leq t \leq \sigma_{1}$, we have for $\sigma_{1} \leq t \leq t_{1}$,

$$
u(x, t)-\tilde{u}(x, t)=a^{2} \int_{\sigma_{1}}^{t} G(x, t ; b, \tau)(f(u(b, \tau))-f(\tilde{u}(b, \tau))) d \tau,
$$

from which we obtain

$$
\Theta \leq a^{2} f^{\prime}(M) k_{1} k_{2} b^{-q / 4}\left[\sum_{i=1}^{\infty} \lambda_{i}^{-3 / 4}\left(1-e^{-\lambda_{i}\left(t-\sigma_{1}\right)}\right)\right] \Theta .
$$

It follows from (7) that for $t \in\left[\sigma_{1}, \min \left\{2 \sigma_{1}, t_{1}\right\}\right]$,

$$
a^{2} f^{\prime}(M) k_{1} k_{2} b^{-q / 4}\left[\sum_{i=1}^{\infty} \lambda_{i}^{-3 / 4}\left(1-e^{-\lambda_{i}\left(t-\sigma_{1}\right)}\right)\right]<1
$$


This gives a contradiction. Thus, we have uniqueness of a solution for $0 \leq t \leq$ $\min \left\{2 \sigma_{1}, t_{1}\right\}$. By proceeding in this way, the integral equation (3) has a unique nonnegative solution $u$ for $0 \leq t \leq t_{1}$.

From Lemma $1(\mathrm{~h})$ and (4), each $u_{n}$ is continuous. To show that $u$ is continuous, we note that

$$
u_{n+1}(x, t)-u_{n}(x, t)=a^{2} \int_{0}^{t} G(x, t ; b, \tau)\left[f\left(u_{n}(b, \tau)\right)-f\left(u_{n-1}(b, \tau)\right)\right] d \tau .
$$

Let $S_{n}=\max _{\bar{D} \times\left[0, t_{1}\right]}\left(u_{n}-u_{n-1}\right)$. Using the Mean Value Theorem, we have

$$
f\left(u_{n}(b, \tau)\right)-f\left(u_{n-1}(b, \tau)\right) \leq f^{\prime}(M) S_{n} .
$$

As in the derivation of $(6)$, we obtain for $0 \leq t \leq \sigma_{1}$,

$$
S_{n+1} \leq a^{2} f^{\prime}(M) k_{1} k_{2} b^{-q / 4}\left[\sum_{i=1}^{\infty} \lambda_{i}^{-3 / 4}\left(1-e^{-\lambda_{i} t}\right)\right] S_{n} .
$$

By (7), the sequence $\left\{u_{n}\right\}$ converges uniformly to $u$ with respect to $x \in \bar{D}$ and $t \in\left[0, \sigma_{1}\right]$. Thus, the integral equation (3) has a unique nonnegative continuous solution $u$ for $0 \leq$ $t \leq \sigma_{1}$. From Lemma 1(a) and 1(b), the integrand of the first term on the right-hand side of (8) is bounded by $f(M) k_{1} k_{2} b^{-q / 4} \sum_{i=1}^{\infty} \lambda_{i}^{1 / 4} e^{-\lambda_{i}(t-\tau)}$, which is integrable for $t>\sigma_{1}$. Thus, if $\sigma_{1}<t_{1}$, then for $\sigma_{1} \leq t \leq t_{1}$, the first term on the right-hand side of (8) is continuous. Let

$$
z(x, t)=a^{2} \int_{\sigma_{1}}^{t} G(x, t ; b, \tau) f(z(b, \tau)) d \tau .
$$

From (8), $z<M$. For $\sigma_{1} \leq t \leq t_{1}$, let us construct a sequence $\left\{z_{i}\right\}$ by $z_{0}(x, t) \equiv 0$, and for $i=0,1,2, \ldots$,

$$
z_{i+1}(x, t)=a^{2} \int_{\sigma_{1}}^{t} G(x, t ; b, \tau) f\left(z_{i}(b, \tau)\right) d \tau .
$$

By Lemma $1(\mathrm{~h})$, each $z_{n}$ is continuous. We have

$$
z_{n+1}(x, t)-z_{n}(x, t)=a^{2} \int_{\sigma_{1}}^{t} G(x, t ; b, \tau)\left(f\left(z_{n}(b, \tau)\right)-f\left(z_{n-1}(b, \tau)\right)\right) d \tau .
$$

Let $Z_{n}=\max _{\bar{D} \times\left[\sigma_{1}, \min \left\{2 \sigma_{1}, t_{1}\right\}\right]}\left|z_{n}-z_{n-1}\right|$. As in the derivation of (9),

$$
Z_{n+1} \leq a^{2} f^{\prime}(M) k_{1} k_{2} b^{-q / 4}\left[\sum_{i=1}^{\infty} \lambda_{i}^{-3 / 4}\left(1-e^{-\lambda_{i}\left(t-\sigma_{1}\right)}\right)\right] Z_{n} .
$$

From (10), the sequence $\left\{z_{n}\right\}$ converges uniformly to $z$, and hence, $z$ is continuous for $\sigma_{1} \leq t \leq \min \left\{2 \sigma_{1}, t_{1}\right\}$. From (8), $u$ is continuous for $\sigma_{1} \leq t \leq \min \left\{2 \sigma_{1}, t_{1}\right\}$. If $2 \sigma_{1}<t_{1}$, then for $2 \sigma_{1} \leq t \leq t_{1}$,

$$
u(x, t)=a^{2} \int_{0}^{2 \sigma_{1}} G(x, t ; b, \tau) f(u(b, \tau)) d \tau+a^{2} \int_{2 \sigma_{1}}^{t} G(x, t ; b, \tau) f(u(b, \tau)) d \tau .
$$

Since the first term on the right-hand side is continuous, we consider the second term. An argument analogous to the above shows that $u$ is continuous for $0 \leq t \leq \min \left\{3 \sigma_{1}, t_{1}\right\}$. By proceeding in this way, the integral equation (3) has a unique nonnegative continuous solution $u$ for $0 \leq t \leq t_{1}$. 
Let $t_{q}$ be the supremum of all $t_{1}$, where $\left[0, t_{1}\right]$ is the interval for which the integral equation (3) has a unique nonnegative continuous solution $u$. If $t_{q}$ is finite and $u$ does not reach $c^{-}$at $t_{q}$, then given any number between $\max _{\bar{D}} u\left(x, t_{q}\right)$ and $c$, a proof similar to the above shows that there exists some $t_{2}>t_{q}$ such that the integral equation (3) has a unique nonnegative continuous solution $u$ for $0 \leq t \leq t_{2}$. This contradicts the definition of $t_{q}$. Hence, if $t_{q}$ is finite, then $u$ reaches $c^{-}$at $t_{q}$.

It follows from $u_{n}$ being an increasing function of $t$ in $D$ that $u$ is a nondecreasing function of $t$. Let $\sigma=\tau-\epsilon$. We have

$$
\begin{aligned}
& a^{2} \int_{0}^{t+\epsilon} G(x, t+\epsilon ; b, \tau) f(u(b, \tau)) d \tau \\
& =a^{2}\left(\int_{0}^{\epsilon} G(x, t+\epsilon ; b, \tau) f(u(b, \tau)) d \tau+\int_{0}^{t} G(x, t ; b, \sigma) f(u(b, \sigma+\epsilon)) d \sigma\right) \\
& \geq a^{2}\left(\int_{0}^{\epsilon} G(x, t+\epsilon ; b, \tau) f(u(b, \tau)) d \tau+\int_{0}^{t} G(x, t ; b, \sigma) f(u(b, \sigma)) d \sigma\right)
\end{aligned}
$$

since $f$ is an increasing function. Thus,

$$
u(x, t+\epsilon)-u(x, t) \geq a^{2} \int_{0}^{\epsilon} G(x, t+\epsilon ; b, \tau) f(u(b, \tau)) d \tau>0 .
$$

Hence, $u$ is a strictly increasing function of $t$ in $D$.

We modify the method of proving Theorem 2.5 of Chan and Tian [6] to show that the solution of the integral equation (3) is actually the solution of the problem (2).

Theorem 2. Before quenching occurs, the problem (2) has a unique nonnegative solution $u$. If $t_{q}$ is finite, then $u$ quenches at $t_{q}$.

Proof. It follows from Lemma $1(\mathrm{~h})$ that for any $t_{3} \in(0, t)$,

$$
\begin{aligned}
& \int_{0}^{t} G(x, t ; b, \tau) f(u(b, \tau)) d \tau \\
& =\lim _{n \rightarrow \infty} \int_{0}^{t-1 / n} G(x, t ; b, \tau) f(u(b, \tau)) d \tau \\
& =\lim _{n \rightarrow \infty} \int_{t_{3}}^{t} \frac{\partial}{\partial \zeta}\left(\int_{0}^{\zeta-1 / n} G(x, \zeta ; b, \tau) f(u(b, \tau)) d \tau\right) d \zeta \\
& \quad+\lim _{n \rightarrow \infty} \int_{0}^{t_{3}-1 / n} G\left(x, t_{3} ; b, \tau\right) f(u(b, \tau)) d \tau .
\end{aligned}
$$

For $\zeta-\tau \geq 1 / n$, it follows from Lemma $1(\mathrm{f})$ and $1(\mathrm{~b})$ that for any $x \in \bar{D}$,

$$
\begin{aligned}
G_{\zeta}(x, \zeta ; b, \tau) f(u(b, \tau)) & \leq f(u(b, \tau))\left(\sum_{i=1}^{\infty}\left|\phi_{i}(x)\right|\left|\phi_{i}(b)\right| \lambda_{i} e^{-\lambda_{i}(\zeta-\tau)}\right) \\
& \leq k_{2}^{2} f(u(b, \tau)) \sum_{i=1}^{\infty} \lambda_{i}^{3 / 2} e^{-\lambda_{i} / n}
\end{aligned}
$$


which is integrable with respect to $\tau$ over $(0, \zeta-1 / n)$. Using the Leibnitz rule (cf. Stromberg [11, p. 380]), we have

$$
\begin{aligned}
& \frac{\partial}{\partial \zeta}\left(\int_{0}^{\zeta-1 / n} G(x, \zeta ; b, \tau) f(u(b, \tau)) d \tau\right) \\
& =G\left(x, \zeta ; b, \zeta-\frac{1}{n}\right) f\left(u\left(b, \zeta-\frac{1}{n}\right)\right)+\int_{0}^{\zeta-1 / n} G_{\zeta}(x, \zeta ; b, \tau) f(u(b, \tau)) d \tau .
\end{aligned}
$$

Let us consider the problem

$$
\begin{gathered}
L w=0 \text { for } x \text { and } \xi \text { in } D, 0<\tau<t, \\
w(0, t ; \xi, \tau)=w(1, t ; \xi, \tau)=0 \text { for } 0<\tau<t, \\
\lim _{t \rightarrow \tau^{+}} x^{q} w(x, t ; \xi, \tau)=\delta(x-\xi) .
\end{gathered}
$$

Using Green's second identity, we obtain for $t \geq \tau$,

$$
\begin{aligned}
w(x, t ; \xi, \tau) & =\int_{D} y^{q} G(x, t ; y, \tau) y^{-q} \delta(y-\xi) d y \\
& =G(x, t ; \xi, \tau) .
\end{aligned}
$$

It follows that $\lim _{t \rightarrow \tau^{+}} x^{q} G(x, t ; \xi, \tau)=\delta(x-\xi)$.

Since $f$ is an increasing function, and $G(x, \zeta ; b, \zeta-1 / n)=G(x, 1 / n ; b, 0)$, which is independent of $\zeta$, we have

$$
\begin{aligned}
& \int_{0}^{t} x^{q} G(x, t ; b, \tau) f(u(b, \tau)) d \tau \\
& =\delta(x-b) \int_{t_{3}}^{t} f(u(b, \zeta)) d \zeta+\lim _{n \rightarrow \infty} \int_{t_{3}}^{t} \int_{0}^{\zeta-1 / n} x^{q} G_{\zeta}(x, \zeta ; b, \tau) f(u(b, \tau)) d \tau d \zeta \\
& +\int_{0}^{t_{3}} x^{q} G\left(x, t_{3} ; b, \tau\right) f(u(b, \tau)) d \tau
\end{aligned}
$$

Let

$$
g_{n}(x, \zeta)=\int_{0}^{\zeta-1 / n} x^{q} G_{\zeta}(x, \zeta ; b, \tau) f(u(b, \tau)) d \tau .
$$

Without loss of generality, let $n>l$. We have

$$
g_{n}(x, \zeta)-g_{l}(x, \zeta)=\int_{\zeta-1 / l}^{\zeta-1 / n} x^{q} G_{\zeta}(x, \zeta ; b, \tau) f(u(b, \tau)) d \tau
$$

Since $x^{q} G_{\zeta}(x, \zeta ; b, \tau) \in C(\bar{D} \times(\tau, T])$ and $f(u(b, \tau))$ is an increasing function of $\tau$, it follows from the Second Mean Value Theorem for Integrals (cf. Stromberg [11, p. 328]) that for any $x \neq b$ and any $\zeta$ in any compact subset of $\left(0, t_{q}\right)$, there exists some real number $\nu$ such that $\zeta-\nu \in(\zeta-1 / l, \zeta-1 / n)$ and

$$
\begin{aligned}
g_{n}(x, \zeta)-g_{l}(x, \zeta) & =f\left(u\left(b, \zeta-\frac{1}{l}\right)\right) \int_{\zeta-1 / l}^{\zeta-\nu} x^{q} G_{\zeta}(x, \zeta ; b, \tau) d \tau \\
& +f\left(u\left(b, \zeta-\frac{1}{n}\right)\right) \int_{\zeta-\nu}^{\zeta-1 / n} x^{q} G_{\zeta}(x, \zeta ; b, \tau) d \tau .
\end{aligned}
$$


From $G_{\zeta}(x, \zeta ; b, \tau)=-G_{\tau}(x, \zeta ; b, \tau)$, we have

$$
\begin{aligned}
g_{n}(x, \zeta)-g_{l}(x, \zeta) & =\left[f\left(u\left(b, \zeta-\frac{1}{n}\right)\right)-f\left(u\left(b, \zeta-\frac{1}{l}\right)\right)\right] x^{q} G(x, \zeta ; b, \zeta-\nu) \\
& +f\left(u\left(b, \zeta-\frac{1}{l}\right)\right) x^{q} G\left(x, \zeta ; b, \zeta-\frac{1}{l}\right) \\
& -f\left(u\left(b, \zeta-\frac{1}{n}\right)\right) x^{q} G\left(x, \zeta ; b, \zeta-\frac{1}{n}\right) .
\end{aligned}
$$

Since for $x \neq b, x^{q} G(x, \zeta ; b, \zeta-\epsilon)=x^{q} G(x, \epsilon ; b, 0)$, which converges to 0 uniformly with respect to $\zeta$ as $\epsilon \rightarrow 0$, it follows that for $x \neq b,\left\{g_{n}\right\}$ is a Cauchy sequence, and hence $\left\{g_{n}\right\}$ converges uniformly with respect to $\zeta$ in any compact subset of $\left(0, t_{q}\right)$. Hence for $x \neq b$,

$$
\begin{aligned}
& \lim _{n \rightarrow \infty} \int_{t_{3}}^{t} \int_{0}^{\zeta-1 / n} x^{q} G_{\zeta}(x, \zeta ; b, \tau) f(u(b, \tau)) d \tau d \zeta \\
& =\int_{t_{3}}^{t} \lim _{n \rightarrow \infty} \int_{0}^{\zeta-1 / n} x^{q} G_{\zeta}(x, \zeta ; b, \tau) f(u(b, \tau)) d \tau d \zeta \\
& =\int_{t_{3}}^{t} \int_{0}^{\zeta} x^{q} G_{\zeta}(x, \zeta ; b, \tau) f(u(b, \tau)) d \tau d \zeta
\end{aligned}
$$

For $x=b$,

$$
-G_{\zeta}(b, \zeta ; b, \tau) f(u(b, \tau))=\sum_{i=1}^{\infty} \phi_{i}^{2}(b) \lambda_{i} e^{-\lambda_{i}(\zeta-\tau)} f(u(b, \tau))
$$

which is positive. Thus, $\left\{-g_{n}\right\}$ is a nondecreasing sequence of nonnegative functions with respect to $\zeta$. By the Monotone Convergence Theorem,

$$
\lim _{n \rightarrow \infty} \int_{t_{3}}^{t} \int_{0}^{\zeta-1 / n} b^{q} G_{\zeta}(b, \zeta ; b, \tau) f(u(b, \tau)) d \tau d \zeta=\int_{t_{3}}^{t} \int_{0}^{\zeta} b^{q} G_{\zeta}(b, \zeta ; b, \tau) f(u(b, \tau)) d \tau d \zeta
$$

Thus from (11),

$$
\frac{\partial}{\partial t} \int_{0}^{t} x^{q} G(x, t ; b, \tau) f(u(b, \tau)) d \tau=\delta(x-b) f(u(b, t))+\int_{0}^{t} x^{q} G_{t}(x, t ; b, \tau) f(u(b, \tau)) d \tau .
$$

By Lemma 1(b) and 1(c),

$$
\begin{aligned}
\left|\sum_{i=1}^{\infty} \frac{\partial}{\partial x} \phi_{i}(x) \phi_{i}(\xi) e^{-\lambda_{i}(t-\tau)}\right| & \leq \sum_{i=1}^{\infty}\left|\phi_{i}^{\prime}(x)\right|\left|\phi_{i}(\xi)\right| e^{-\lambda_{i}(t-\tau)} \\
& \leq k_{2} k_{3} \sum_{i=1}^{\infty} \lambda_{i}^{3 / 4} e^{-\lambda_{i}(t-\tau)}
\end{aligned}
$$

which converges uniformly with respect to $x$ in any compact subset of $(0,1]$ with $t-\tau \geq \epsilon$. By Lemma $1(\mathrm{~g})$ and the Leibnitz rule, we have for any $x$ in any compact subset of $(0,1]$ and $t$ in any compact subset of $\left(0, t_{q}\right)$,

$$
\frac{\partial}{\partial x} \int_{0}^{t-\epsilon} G(x, t ; b, \tau) f(u(b, \tau)) d \tau=\int_{0}^{t-\epsilon} G_{x}(x, t ; b, \tau) f(u(b, \tau)) d \tau .
$$


Since $\phi_{i}$ is an eigenfunction, it follows from Lemma 1(b) that

$$
\begin{aligned}
\left|\sum_{i=1}^{\infty} \frac{\partial^{2}}{\partial x^{2}} \phi_{i}(x) \phi_{i}(\xi) e^{-\lambda_{i}(t-\tau)}\right| & \leq \sum_{i=1}^{\infty}\left|\phi_{i}^{\prime \prime}(x)\right|\left|\phi_{i}(\xi)\right| e^{-\lambda_{i}(t-\tau)} \\
& =\sum_{i=1}^{\infty} \lambda_{i} x^{q}\left|\phi_{i}(x)\right|\left|\phi_{i}(\xi)\right| e^{-\lambda_{i}(t-\tau)} \\
& \leq k_{2}^{2} \sum_{i=1}^{\infty} \lambda_{i}^{3 / 2} e^{-\lambda_{i}(t-\tau)}
\end{aligned}
$$

which converges uniformly with respect to $x$ in any compact subset of $(0,1]$ with $t-\tau \geq \epsilon$. By Lemma $1(\mathrm{~g})$ and the Leibnitz rule, we have for any $x$ in any compact subset of $(0,1]$ and $t$ in any compact subset of $\left(0, t_{q}\right)$,

$$
\frac{\partial}{\partial x} \int_{0}^{t-\epsilon} G_{x}(x, t ; b, \tau) f(u(b, \tau)) d \tau=\int_{0}^{t-\epsilon} G_{x x}(x, t ; b, \tau) f(u(b, \tau)) d \tau .
$$

For any $x_{1} \in D$,

$$
\begin{aligned}
& \lim _{\epsilon \rightarrow 0} \int_{0}^{t-\epsilon} G(x, t ; b, \tau) f(u(b, \tau)) d \tau \\
& =\lim _{\epsilon \rightarrow 0} \int_{x_{1}}^{x}\left(\frac{\partial}{\partial \eta} \int_{0}^{t-\epsilon} G(\eta, t ; b, \tau) f(u(b, \tau)) d \tau\right) d \eta+\lim _{\epsilon \rightarrow 0} \int_{0}^{t-\epsilon} G\left(x_{1}, t ; b, \tau\right) f(u(b, \tau)) d \tau \\
& =\lim _{\epsilon \rightarrow 0} \int_{x_{1}}^{x} \int_{0}^{t-\epsilon} G_{\eta}(\eta, t ; b, \tau) f(u(b, \tau)) d \tau d \eta+\int_{0}^{t} G\left(x_{1}, t ; b, \tau\right) f(u(b, \tau)) d \tau
\end{aligned}
$$

By the Fubini Theorem (cf. Stromberg [11, p. 352]),

$$
\begin{aligned}
\lim _{\epsilon \rightarrow 0} \int_{x_{1}}^{x} \int_{0}^{t-\epsilon} G_{\eta}(\eta, t ; b, \tau) f(u(b, \tau)) d \tau d \eta & =\lim _{\epsilon \rightarrow 0} \int_{0}^{t-\epsilon}\left(f(u(b, \tau)) \int_{x_{1}}^{x} G_{\eta}(\eta, t ; b, \tau) d \eta\right) d \tau \\
& =\lim _{\epsilon \rightarrow 0} \int_{0}^{t-\epsilon} f(u(b, \tau))\left(G(x, t ; b, \tau)-G\left(x_{1}, t ; b, \tau\right)\right) d \tau \\
& =\int_{0}^{t} f(u(b, \tau))\left(G(x, t ; b, \tau)-G\left(x_{1}, t ; b, \tau\right)\right) d \tau,
\end{aligned}
$$

which exists by Lemma 1(h). Therefore,

$$
\begin{aligned}
\int_{0}^{t} f(u(b, \tau))\left(G(x, t ; b, \tau)-G\left(x_{1}, t ; b, \tau\right)\right) d \tau & =\int_{0}^{t} f(u(b, \tau))\left(\int_{x_{1}}^{x} G_{\eta}(\eta, t ; b, \tau) d \eta\right) d \tau \\
& =\int_{x_{1}}^{x} \int_{0}^{t} G_{\eta}(\eta, t ; b, \tau) f(u(b, \tau)) d \tau d \eta
\end{aligned}
$$

From (12),

$$
\begin{aligned}
& \int_{0}^{t} G(x, t ; b, \tau) f(u(b, \tau)) d \tau \\
& =\int_{x_{1}}^{x} \int_{0}^{t} G_{\eta}(\eta, t ; b, \tau) f(u(b, \tau)) d \tau d \eta+\int_{0}^{t} G\left(x_{1}, t ; b, \tau\right) f(u(b, \tau)) d \tau .
\end{aligned}
$$


Thus,

$$
\frac{\partial}{\partial x} \int_{0}^{t} G(x, t ; b, \tau) f(u(b, \tau)) d \tau=\int_{0}^{t} G_{x}(x, t ; b, \tau) f(u(b, \tau)) d \tau
$$

For any $x_{2} \in D$,

$$
\begin{aligned}
& \lim _{\epsilon \rightarrow 0} \int_{0}^{t-\epsilon} G_{x}(x, t ; b, \tau) f(u(b, \tau)) d \tau \\
& =\lim _{\epsilon \rightarrow 0} \int_{x_{2}}^{x}\left(\frac{\partial}{\partial \eta} \int_{0}^{t-\epsilon} G_{\eta}(\eta, t ; b, \tau) f(u(b, \tau)) d \tau\right) d \eta+\lim _{\epsilon \rightarrow 0} \int_{0}^{t-\epsilon} G_{\eta}\left(x_{2}, t ; b, \tau\right) f(u(b, \tau)) d \tau \\
& =\lim _{\epsilon \rightarrow 0} \int_{x_{2}}^{x} \int_{0}^{t-\epsilon} G_{\eta \eta}(\eta, t ; b, \tau) f(u(b, \tau)) d \tau d \eta+\int_{0}^{t} G_{\eta}\left(x_{2}, t ; b, \tau\right) f(u(b, \tau)) d \tau .
\end{aligned}
$$

By the Fubini Theorem,

$$
\begin{aligned}
\lim _{\epsilon \rightarrow 0} \int_{x_{2}}^{x} \int_{0}^{t-\epsilon} G_{\eta \eta}(\eta, t ; b, \tau) f(u(b, \tau)) d \tau d \eta & =\lim _{\epsilon \rightarrow 0} \int_{0}^{t-\epsilon}\left(f(u(b, \tau)) \int_{x_{2}}^{x} G_{\eta \eta}(\eta, t ; b, \tau) d \eta\right) d \tau \\
& =\lim _{\epsilon \rightarrow 0} \int_{0}^{t-\epsilon} f(u(b, \tau))\left(G_{\eta}(x, t ; b, \tau)-G_{\eta}\left(x_{2}, t ; b, \tau\right)\right) d \tau \\
& =\int_{0}^{t} f(u(b, \tau))\left(G_{\eta}(x, t ; b, \tau)-G_{\eta}\left(x_{2}, t ; b, \tau\right)\right) d \tau
\end{aligned}
$$

which exists by (13). Therefore,

$$
\int_{0}^{t} f(u(b, \tau))\left(G_{\eta}(x, t ; b, \tau)-G_{\eta}\left(x_{2}, t ; b, \tau\right)\right) d \tau=\int_{x_{2}}^{x} \int_{0}^{t} G_{\eta \eta}(\eta, t ; b, \tau) f(u(b, \tau)) d \tau d \eta
$$

From (14),

$$
\begin{aligned}
& \int_{0}^{t} G_{x}(x, t ; b, \tau) f(u(b, \tau)) d \tau \\
& =\int_{x_{2}}^{x} \int_{0}^{t} G_{\eta \eta}(\eta, t ; b, \tau) f(u(b, \tau)) d \tau d \eta+\int_{0}^{t} G_{\eta}\left(x_{2}, t ; b, \tau\right) f(u(b, \tau)) d \tau
\end{aligned}
$$

Thus,

$$
\frac{\partial}{\partial x} \int_{0}^{t} G_{x}(x, t ; b, \tau) f(u(b, \tau)) d \tau=\int_{0}^{t} G_{x x}(x, t ; b, \tau) f(u(b, \tau)) d \tau
$$

It follows from (13) that for any $x$ in any compact subset of $D$ and any $t$ in any compact subset of $\left(0, t_{q}\right)$,

$$
\frac{\partial^{2}}{\partial x^{2}} \int_{0}^{t} G(x, t ; b, \tau) f(u(b, \tau)) d \tau=\int_{0}^{t} G_{x x}(x, t ; b, \tau) f(u(b, \tau)) d \tau .
$$

Before quenching occurs, it follows from the integral equation (3) that for $x \in D$ and $t>0$,

$$
\begin{aligned}
L u & =a^{2} \delta(x-b) f(u(b, t))+a^{2} \int_{0}^{t} L G(x, t ; b, \tau) f(u(b, \tau)) d \tau \\
& =a^{2} \delta(x-b) f(u(b, t))+a^{2} \delta(x-b) \lim _{\epsilon \rightarrow 0} \int_{0}^{t-\epsilon} \delta(t-\tau) f(u(b, \tau)) d \tau \\
& =a^{2} \delta(x-b) f(u(x, t)) .
\end{aligned}
$$


From $(3), \lim _{t \rightarrow 0} u(x, t)=0$ for $x \in \bar{D}$. Since $G(0, t ; \xi, \tau)=G(1, t ; \xi, \tau)=0$, we have $u(0, t)=u(1, t)=0$. Thus, the nonnegative continuous solution of the integral equation (3) is a solution of the problem (2). Since a solution of the problem (2) is a solution of the integral equation (3), which has a unique solution before quenching occurs, $u$ is the unique solution of the problem (2).

If $t_{q}$ is finite, then by Theorem $1, u$ reaches $c^{-}$at $t_{q}$. Thus, $u$ quenches at $t_{q}$.

Our next result shows that $b$ is the single quenching point.

TheOREM 3. The solution $u(x, t)$ of the problem $(2)$ attains its maximum at $(b, t)$. If $u$ quenches, then $b$ is the single quenching point.

Proof. Since $u(b, t)$ is known, let us consider the following problems:

$$
\left.\begin{array}{c}
\left.\begin{array}{l}
L u=0 \text { in }(0, b) \times\left(0, t_{q}\right), \\
u(x, 0)=0 \text { for } 0 \leq x \leq b, u(0, t)=0 \text { and } u(b, t)=u(b, t) \text { for } 0<t<t_{q},
\end{array}\right\} \\
L u=0 \text { in }(b, 1) \times\left(0, t_{q}\right), \\
u(x, 0)=0 \text { for } b \leq x \leq 1, u(b, t)=u(b, t) \text { and } u(1, t)=0 \text { for } 0<t<t_{q} .
\end{array}\right\}
$$

By the weak maximum principle (cf. Friedman [8, p. 39]), $u$ attains its maximum at $b$ for each of the problems (15) and (16). Since $u$ is a strictly increasing function of $t$ in $D, u(x, t)$ attains its maximum at $(b, t)$.

If $u$ quenches, then it quenches at $b$. For the problem (15), it follows from the parabolic version of Hopf's Lemma (cf. Friedman [8, p. 49]) that for any fixed $t \in\left(0, t_{q}\right), u_{x}(0, t)>$ 0 . For any $x \in(0, b), u_{x x}=x^{q} u_{t}$, which is nonnegative by Theorem 1 . Hence, $u$ is concave up. Similarly for any fixed $t \in\left(0, t_{q}\right), u_{x}(1, t)<0$. For any $x \in(b, 1), u_{x x}=x^{q} u_{t} \geq 0$, and hence, $u$ is concave up. Thus if $u$ quenches, then $b$ is the single quenching point.

3. Critical length and no quenching in infinite time. We modify the proof of Theorem 3 of Chan and Kaper [2] to obtain the following result.

THEOREM 4. If there exists a constant $C \in(0, c)$ such that $\lim _{t \rightarrow \infty} u(x, t) \leq C$, then $u(x, t)$ converges uniformly on $\bar{D}$ from below to a solution $U(x)$ of the nonlinear two-point boundary value problem,

$$
-U^{\prime \prime}(x)=a^{2} \delta(x-b) f(U(x)) \text { in } D, U(0)=U(1)=0 .
$$

Furthermore, $u(x, t)<U(x)$ in $D \times(0, \infty)$.

Proof. Since the homogeneous problem corresponding to (17) has only the trivial solution, its Green's function $g(x ; \xi)$ exists. A direct computation gives

$$
g(x ; \xi)= \begin{cases}\xi(1-x), & 0 \leq \xi \leq x \\ x(1-\xi), & x<\xi \leq 1\end{cases}
$$

Let

$$
F(x, t)=\int_{D} g(x ; \xi) \xi^{q} u(\xi, t) d \xi
$$


which is bounded, because the integrand is bounded. Since $u$ is the solution of the problem (2), $F(x, t)$ may be regarded as a distribution. By Green's second identity,

$$
\begin{aligned}
F_{t}(x, t) & =\int_{D} g(x ; \xi)\left(u_{\xi \xi}(\xi, t)+a^{2} \delta(\xi-b) f(u(\xi, t))\right) d \xi \\
& =-u(x, t)+a^{2} g(x ; b) f(u(b, t)) .
\end{aligned}
$$

Since $u$ is a strictly increasing function of $t$ in $D$ and $f(u)$ is strictly increasing, the term $a^{2} g(x ; b) f(u(b, t))$ in $(18)$ is monotone increasing with respect to $t$. It follows from the continuity of $f$ that

$$
\lim _{t \rightarrow \infty} F_{t}(x, t)=-\lim _{t \rightarrow \infty} u(x, t)+a^{2} g(x ; b) f\left(\lim _{t \rightarrow \infty} u(b, t)\right)
$$

which exists since $u \leq C$. We note that $F$ is a strictly increasing function of $t$ in $D$. If $\lim _{t \rightarrow \infty} F_{t}(x, t)$ were strictly positive at some point $x$, then $F(x, t)$ would increase without bound as $t$ tends to infinity. Thus, $\lim _{t \rightarrow \infty} F_{t}(x, t)=0$. It follows from (19) that

$$
\tilde{U}(x)=a^{2} g(x ; b) f(\tilde{U}(b)),
$$

where $\tilde{U}(x)=\lim _{t \rightarrow \infty} u(x, t)$. Since $u$ is a strictly increasing function of $t$ in $D$, we have $u(x, t)<\tilde{U}(x)$ in $D \times(0, \infty)$. By a direct differentiation,

$$
\tilde{U}^{\prime \prime}(x)=-a^{2} \delta(x-b) f(\tilde{U}(b))=-a^{2} \delta(x-b) f(\tilde{U}(x)) .
$$

From $(20), \tilde{U}(0)=\tilde{U}(1)=0$. Thus, $\tilde{U}(x)$ is a solution of the problem (17). Since $\tilde{U}$ is continuous, the uniform convergence of $u$ to $\tilde{U}$ on $\bar{D}$ follows from the Dini Theorem. The theorem is then proved.

Let $v$ denote the solution of the problem (1) with $a$ and $\beta$ being replaced by $a+\alpha$ and $\beta(a+\alpha) / a$ respectively for some constant $\alpha>0$. Let $\varsigma=(a+\alpha) x, \gamma=(a+\alpha)^{q+2} t$, $\beta=(a+\alpha) b, F(u(\varsigma, \gamma))=f(v(x, t)), T_{\alpha}=\rho /(a+\alpha)^{q+2}$, and $\Omega_{\alpha}=D \times\left(0, T_{\alpha}\right]$. Then,

$$
\left.\begin{array}{c}
L v=(a+\alpha)^{2} \delta(x-b) f(v(x, t)) \text { in } \Omega_{\alpha}, \\
v(x, 0)=0 \text { on } \bar{D}, v(0, t)=v(1, t)=0 \text { for } 0<t \leq T_{\alpha} .
\end{array}\right\}
$$

THEOREM 5. In $\Omega_{\alpha}, v(x, t)>u(x, t)$.

Proof. Let us construct two sequences $\left\{v_{i}(x, t)\right\}$ and $\left\{u_{i}(x, t)\right\}$ in $\Omega_{\alpha}$ by $v_{0}(x, t)=$ $u_{0}(x, t)=0$, and for $i=0,1,2, \ldots$,

$$
\begin{gathered}
L v_{i+1}=(a+\alpha)^{2} \delta(x-b) f\left(v_{i}(x, t)\right) \text { in } \Omega_{\alpha}, \\
v_{i+1}(x, 0)=0 \text { on } \bar{D}, v_{i+1}(0, t)=v_{i+1}(1, t)=0 \text { for } 0<t \leq T_{\alpha} ; \\
L u_{i+1}=a^{2} \delta(x-b) f\left(u_{i}(x, t)\right) \text { in } \Omega_{\alpha}, \\
u_{i+1}(x, 0)=0 \text { on } \bar{D}, u_{i+1}(0, t)=u_{i+1}(1, t)=0 \text { for } 0<t \leq T_{\alpha} .
\end{gathered}
$$

By (3),

$$
v_{1}(x, t)-u_{1}(x, t)=\left[(a+\alpha)^{2}-a^{2}\right] f(0) \int_{0}^{t} G(x, t ; b, \tau) d \tau>0 .
$$

Let us assume that for some positive integer $j, v_{j}(x, t)>u_{j}(x, t)$. Then,

$$
v_{j+1}(x, t)-u_{j+1}(x, t)>a^{2} \int_{0}^{t} G(x, t ; b, \tau)\left(f\left(v_{j}(b, \tau)\right)-f\left(u_{j}(b, \tau)\right)\right) d \tau>0 .
$$


By the principle of mathematical induction, $v_{n}(x, t)>u_{n}(x, t)$ for all positive integers $n$. A proof similar to those of Theorems 1 and 2 shows that $v(x, t)=\lim _{n \rightarrow \infty} v_{n}(x, t)$ is the solution of the problem (21). Hence, $v(x, t) \geq u(x, t)$ in $\Omega_{\alpha}$.

By (3),

$$
v(x, t)-u(x, t)>a^{2} \int_{0}^{t} G(x, t ; b, \tau)(f(v(b, \tau))-f(u(b, \tau))) d \tau \geq 0 .
$$

The theorem is then proved.

Theorem 6. If $\lim _{t \rightarrow s} u(b, t)=c^{-}$, where $s \leq \infty$, then $v(b, t)$ quenches in a finite time.

Proof. From (3),

$$
\lim _{t \rightarrow s} \int_{0}^{t} G(b, t ; b, \tau) f(u(b, \tau)) d \tau=\frac{c}{a^{2}} .
$$

Since in $D, u$ and $v$ are strictly increasing functions of $t$ and $v>u$, there exists some $t_{q}$ $(<s)$ such that

$$
\lim _{t \rightarrow t_{q}} \int_{0}^{t} G(b, t ; b, \tau) f(v(b, \tau)) d \tau=\frac{c}{(a+\alpha)^{2}} .
$$

Hence, $v(b, t)$ quenches in a finite time.

Theorems 4 and 5 imply that there exists a critical length $a^{*}$ such that $u$ exists on $\bar{D}$ for all $t>0$ if $a<a^{*}$. The critical length $a^{*}$ is determined as the supremum of all positive values $a$ for which a solution $U$ of (17) exists. Hence, it is the same as that for $q=0$. According to Theorem $6, u$ quenches in a finite time if $a>a^{*}$.

TheOREM 7. The solution $u$ does not quench in infinite time.

Proof. Since $u(x, t)$ attains its maximum at $(b, t), U(x)=\lim _{t \rightarrow \infty} u(x, t)$ attains its maximum at $b$. From (20),

$$
\begin{aligned}
a^{*} & =\max \left(\frac{U(b)}{g(b, b) f(U(b))}\right)^{1 / 2} \\
& =\frac{1}{[b(1-b)]^{1 / 2}} \max \left(\frac{U(b)}{f(U(b))}\right)^{1 / 2} .
\end{aligned}
$$

Let us consider the function

$$
\psi(s)=\frac{s}{f(s)} .
$$

Since $\psi(s)>0$ for $0<s<c$, and $\psi(0)=0=\lim _{s \rightarrow c^{-}} \psi(s)$, a direct computation shows that $\psi(s)$ attains its maximum when $\psi(s)=1 / f^{\prime}(s)$, where $s \in(0, c)$ by Rolle's Theorem. Thus, $\max (U(b) / f(U(b)))^{1 / 2}$ occurs when

$$
\frac{U(b)}{f(U(b))}=\frac{1}{f^{\prime}(U(b))},
$$

where $0<U(b)<c$. This implies that $U(x)$ exists when $a=a^{*}$. Hence for $a \leq a^{*}, u$ exists globally and is uniformly bounded away from $c$. Since $u$ quenches in a finite time for $a>a^{*}, u$ does not quench in infinite time. 
For illustration, let $f(u)=(1-u)^{-p}$. A direct computation shows that $U(b)[1-U(b)]^{p}$ attains its maximum when $U(b)=1 /(1+p)$. Therefore,

$$
a^{*}=\left(\frac{p^{p}}{b(1-b)(1+p)^{1+p}}\right)^{1 / 2} \text {. }
$$

\section{REFERENCES}

[1] C. Y. Chan and W. Y. Chan, Existence of classical solutions for degenerate semilinear parabolic problems, Appl. Math. Comput. 101, 125-149 (1999)

[2] C. Y. Chan and H. G. Kaper, Quenching for semilinear singular parabolic problems, SIAM J. Math Anal 20, 558-566 (1989)

[3] C. Y. Chan and P. C. Kong, Quenching for degenerate semilinear parabolic equations, Appl. Anal. $54,17-25$ (1994)

[4] C. Y. Chan and P. C. Kong, Channel flow of a viscous fluid in the boundary layer, Quart. Appl. Math 55, 51-56 (1997)

[5] C. Y. Chan and H. T. Liu, Does quenching for degenerate parabolic equations occur at the boundaries?, Dynam. Contin. Discrete Impuls. Systems (Series A) 8, 121-128 (2001)

[6] C. Y. Chan and H. Y. Tian, Single-point blow-up for a degenerate parabolic problem due to a concentrated nonlinear source, Quart. Appl. Math. 61, 363-385 (2003).

[7] K. Deng and C. A. Roberts, Quenching for a diffusive equation with a concentrated singularity, Differential Integral Equations 10, 369-379 (1997)

[8] A. Friedman, Partial Differential Equations of Parabolic Type, Prentice-Hall, Englewood Cliffs, NJ, 1964 , pp. 39 and 49

[9] K. E. Gustafson, Introduction to Partial Differential Equations and Hilbert Space Methods, 2nd ed., John Wiley \& Sons, New York, NY, 1987, p. 176

[10] H. L. Royden, Real Analysis, 3rd ed., Macmillan Publishing Co., New York, NY, 1988, p. 87

[11] K. R. Stromberg, An Introduction to Classical Real Analysis, Wadsworth International Group, Belmont, CA, 1981, pp. 328, 352, and 380

[12] G. N. Watson, A Treatise on the Theory of Bessel Functions, 2nd ed., Cambridge University Press, New York, NY, 1958, p. 506 